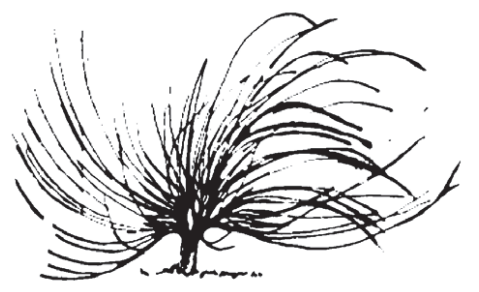

\title{
Tejiendo Ideas: Una Propuesta para la Enseñanza Estratégica del Uso de Conectores en Ensayos Académicos
}

\author{
Paula Alonso Chacón ${ }^{1}$ \\ Universidad Nacional \\ Heredia, Costa Rica \\ paula.alonso.chacon@una.cr
}

\begin{abstract}
Resumen
Este ensayo tiene dos propósitos. El primero es exponer los altibajos discursivos, que presentan los estudiantes universitarios de primer ingreso del Centro de Estudios Generales (CEG) de la Universidad Nacional, respecto del uso de los conectores discursivos en ensayos académicos; esto como secuela de la enseñanza gramatical de la lengua materna. El segundo es compartir el diseño de una propuesta didáctica orientada a la enseñanza estratégica de este recurso lingüístico, a fin de mermar las deficiencias discursivas en torno a la cohesión textual en los cursos de expresión escrita, en el nivel de educación superior. Dichos propósitos constituyen algunos rendimientos del Trabajo Final de Graduación, para optar por el grado de Magister en Educación con énfasis en Docencia Universidad en la Universidad Nacional.
\end{abstract}

Palabras claves: conectores discursivos, enseñanza estratégica, estudiantes universitarios de primer ingreso del CEG, ensayos académicos.

Recibido: 10 de setiembre de 2013 - Aprobado: 20 de noviembre de 2013

1 Filóloga y docente de Centro de Estudios Generales de la UNA. Magister en Educación con énfasis en Docencia Universitaria por la Universidad Nacional de Costa Rica. 


\begin{abstract}
This essay has two purposes. The first one is to show the ups and downs of discourse, presented by freshmen from the Centre for General Studies (CEG) at "Universidad Nacional" in the use of discourse connectors in academic papers as a consequence of the grammatical teaching of the mother tongue. The second one is to share the design of a didactically oriented strategic teaching of this language resource in order to diminish the discursive gaps surrounding textual cohesion in writing courses at the higher education level. These purposes are some of the results from a Final Graduation Project from the Master's Degree Program in Education with an emphasis on University Level Teaching from "Universidad Nacional."
\end{abstract}

Keywords: discourse connectors, strategic teaching, didactic proposal, academic essays, freshmen college students of CEG.

\title{
Introducción
}

\section{La escritura es importante en la escuela porque es importante fuera de la escuela, no al revés.}

E. Ferreiro

\begin{abstract}
A 1 revisar la literatura actualizada, en materia de lingüística, en particular, aquella que versa sobre la enseñanza de la escritura 1 en lengua materna, observo un antagonismo entre dos enfoques: el gramatical y el discursivo. En el primero, la concepción de lengua se homologa a una "bella expresión del pensamiento", materializada a través de un conjunto de reglas morfosintácticas, semánticas y de excepciones, que convergen en el uso "correcto" de la producción oral y escrita (Sánchez, 2004; Silveira, traducido por Matoso, 1999). En el segundo, la lengua se esboza como una práctica social, que se adapta a diversas situaciones comunicativas, por medio de las siguientes adecuaciones lingüísticas: la variación (dialectal o estándar), el registro (general-específico, objetivo-subjetivo), el tipo de texto (informativo, argumentativo, narrativo, descriptivo) y la relación entre los interlocutores (formal-informal) (Cassany, 1999; Calsamiglia y Tusón, 2007).
\end{abstract}


Por ello, el enfoque gramatical se aboca al estudio fragmentado de elementos lingüísticos como la palabra, la frase y la oración, con el propósito de discriminar los usos correctos de los incorrectos; mientras que el enfoque discursivo se basa en la funcionalidad del discurso dentro de un ámbito específico; es decir, las características, la tipología y las secuencias textuales; así como el nivel de especialización del lenguaje, según el medio profesional o social. Asimismo, examina las competencias cognitivas inherentes a todo proceso de redacción, a saber: la generación, la esquematización, la construcción y la reformulación de ideas (Sánchez, 2004).

A partir de esta contextualización, cabe interrogarse ¿Cuál ha sido el enfoque que ha predominado en la enseñanza formal de la expresión escrita, en Costa Rica? La mayoría de las investigaciones sobre el tema revela; por una parte, que el sistema educativo ha optado por una clara inclinación purista del lenguaje, que ha permeado los programas de cursos y los libros de texto; por otra parte, que dicha tendencia constituye la causa del insuficiente dominio del código escrito por parte del estudiantado, a lo largo de la Educación Básica, Diversificada y Superior (Rojas y Umaña;1986; Jiménez, 1987; Quesada y Ramírez, 1993; Garita, 2001 citados por Sánchez, 2006).

Así las cosas, en las páginas siguientes, evidenciaré la importancia del aprendizaje de la escritura formal, desde el estudio de la textualidad. En el caso que nos ocupa, destacaré la cohesión como propiedad textual, a fin de conectar ideas en discursos académicos. Para situar a los lectores no especializados, precisaré qué son los conectores discursivos, cuál es su tipología y sus funciones, según los axiomas de Montolío (2000, 2001). Luego, expondré los altibajos discursivos, que presentan los estudiantes universitarios en lo concerniente al uso de este recurso lingüístico, lo cual justificará la descripción de una propuesta didáctica orientada a la enseñanza estratégica de los conectores, a fin de subsanar algunas deficiencias, en materia discursiva, patentes en los cursos de expresión escrita de nuestras universidades.

\section{Importancia del estudio de la textualidad: La cohesión como pro- piedad textual}

De acuerdo con Cassany (1999), todo texto, sea oral o escrito, constituye una unidad comunicativa, que permite accionar a los interlocutores a través del uso lingüístico determinado. 
Ahora bien, para que una comunidad de hablantes reconozca a un texto como tal; esto es, que opere, de forma eficiente, como mensaje auténtico en una situación comunicativa precisa, debe incluir un grupo de rasgos llamados cualidades o propiedades del texto. Algunos de ellos son: la coherencia (estructuración global del significado del texto), la concisión (uso pertinente de palabras para transmitir una idea), la adecuación lingüística (escogencia de la variedad dialectal, de acuerdo con el contexto) y el repertorio (variedad sintáctica y léxica de un escrito).

Sin embargo, para nuestros efectos, la propiedad trascendental es la cohesión. Esta se define, desde una perspectiva semántica, como una red de conexiones entre los enunciados ${ }^{2}$ de un mismo párrafo y también entre los enunciados de distintos párrafos, de manera razonada. Entonces, en este punto, surgen los conectores del discurso como uno de los mecanismos cohesivos por excelencia.

¿Por qué son tan importantes? Porque el objetivo primordial de estas unidades lingüísticas es garantizarle al lector una adecuada interpretación de la información a la cual se enfrenta al leer, para luego asegurar el entendimiento total del mensaje. Así, se convierten en una suerte de guías para desentrañar el significado existente entre los enunciados, sin lugar a equívocos. De ahí que, acertadamente, Montolio (2001) establezca una metáfora entre la función clarificadora de estos y las señales de tránsito en la carretera:

(...) de la misma manera que las señales viarias indican itinerarios del tipo "curva peligrosa a la derecha", "atención: cambio de rasante" o "dirección obligatoria", las instrucciones que aportan los elementos que estamos analizando son del tipo: "la información que viene a continuación constituye la causa de la que se deduce la que vendrá detrás", "la información que sigue se opone en el razonamiento argumentativo a la anterior", o "atención: ahora llega la conclusión y, por tanto, la información que realmente interesa. (p.21).

En consecuencia, el prescindir de la conexión, el infrautilizarla o utilizarla, de forma inadecuada, supone la aniquilación de la esencia misma del texto, dado que estaríamos ante una lista de enunciados

2 El enunciado se define como una unidad de comunicación intencional vinculada a un contexto determinado (Cervera, A., Hernández, G., Pichardo, C., \& Sánchez, J.: 2006, p. 505). 
ambiguos, o bien, carentes de sentido para la sociedad de hablantes (Cassany, 2008). Además, se exterminaría la autonomía textual, con lo cual nos sobrevendría la Torre de Babel; en otras palabras, una gran confusión que nos obligaría a averiguar a base de ensayo-error las intenciones comunicativas del otro.

\section{Conectores discursivos: Una definición, una tipología y múltiples funciones textuales}

Según Montolío (2001), los conectores discursivos son partículas que evidencian la relación lógico-semántica entre dos o más proposiciones $^{3}$, atinadamente, cuyo objeto es indicarle al lector cuál es el sentido del engranaje de los enunciados; es decir, si estos tienen la misma categoría o si hay una jerarquización entre ellos, mostrando una secuencia expositiva o argumentativa.

Para estudiar los conectores discursivos dentro de los ensayos académicos del estudiantado universitario, adopté la clasificación de Montolío (2001) y de Calsamiglia y Tusón (2007), la cual se estructura en aditivos y organizadores de la información discursiva, contraargumentativos, consecutivos y de finalidad, de modo que siguiendo esta tipología, expondré las funciones de cada uno:

- $\quad$ Conectores aditivos y organizadores de la información discursiva: este tipo de conector posee dos funciones. La primera es introducir información nueva, que conserva la misma temática del enunciado anterior. Por consiguiente, se explicita que la información anterior y posterior al conector aditivo es parte de un mismo bloque significativo. La segunda es organizar la información discursiva, al señalar la estructura de un macroenunciado; así pues, dispone las ideas y las secciona en microenunciados, para que su interpretación sea más fácil. A su vez, dentro de esta función, se encuentran los conectores que abren una serie discursiva, los que presentan enumeraciones relativas al enunciado anterior $\mathrm{y}$, por último, los que cierran una serie discursiva.

- No obstante, el hecho de que todos sean aditivos, no los hace idénticos y, por tanto, intercambiables; de hecho, los organizadores

3 En este trabajo, el concepto de proposición se equipara al de enunciado. 
de la información benefician la progresión discursiva y la distribución de datos, al mostrar un nuevo aspecto del tema, sin jerarquizar los enunciados que engloba.

- $\quad$ Algunos de ellos son: asimismo, igualmente, de iguall mismo modo/ maneralforma, por una parte... por otra parte, por otro lado, por su parte, a su vez.

- $\quad$ Estos se distinguen de los que no solo introducen información novedosa, sino que establecen un procedimiento argumentativo e incluyen la jerarquización de ideas, pues de los enunciados que abarca, uno es superior en relación con el otro, debido a que constituye la introducción del argumento. A continuación, se ejemplifican: además, es más, por añadidura, por lo demás, incluso, inclusive.

- Conectores contraargumentativos: expresan información contrastiva, que vincula los enunciados correspondientes a un solo bloque discursivo. Ellos son: aunque, a pesar de (que), pese a (que), si bien, pero, sin embargo, no obstante, ahora bien, con todo, a pesar de todo, aun asi o de todas formas, mientras que, en tanto que, sino que, en cambio, por el contrario y antes bien, de todas formas, de todas maneras y de todos modos.

- $\quad$ Dentro de estos, pero, sin embargo, no obstante y al contrario introducen argumentos fuertes, desde una óptica argumentativa, con lo cual anulan, semánticamente, los argumentos de las proposiciones anteriores. Por otra parte, los conectores si bien, aunque, a pesar de, aun así, pese a y aun cuando introducen argumentos débiles, pues su significado no es lo bastante contundente, para frustrar la consumación del argumento.

- Conectores consecutivos: las oraciones causales y consecutivas presentan una relación de causa-consecuencia, que atañe a un mismo grupo proposicional. Estas secuencias textuales encierran una misma conexión semántica, en vista de que un fragmento exhibe una causa y el otro la consecuencia. Sin duda, la expresión se aclara mediante el uso del conector.

- $\quad$ Para fines argumentativos, esta relación es imprescindible, pues el argumentar consiste en exponer y verificar cómo desde una premisa se llega a una conclusión.

- Dentro de ellos hay tres subgrupos: los que expresan la consecuencia derivada de una causa (por eso, por ello o por esa/ tal/ 
dicha/razón/ causa/ese motivo, de ahi que, por lo que), los que enfatizan en una causa (porque, ya que, puesto que, visto que, puesto que, dado que, debido a que) y los que enfatizan la consecuencia (en consecuencia, por consiguiente, por tanto).

- $\quad$ Conectores de finalidad: introducen la causa como meta o propósito que persigue el enunciado. Estos son: para (que), a fin de que, con el propósito/ objeto de, de tal modo que.

\section{La conexión inapropiada de ideas en ensayos estudiantiles es habi- tar el país de Babel}

En la introducción de este ensayo les anuncié que les exteriorizaría las vicisitudes discursivas respecto de la conexión en los ensayos académicos de los estudiantes universitarios. Ahora, ustedes se preguntarán, ¿Cuál es la evidencia para sostener dicha tesis? a lo que les contestaré que, en el transcurso de mis estudios de postgrado, realicé una investigación sobre el uso de los conectores discursivos en el Centro de Estudios Generales de la Universidad Nacional, con estudiantes de primer ingreso, provenientes de colegios públicos y residentes de la Gran Área Metropolitana (GAM), con el fin de obtener conclusiones sobre el comportamiento de este recurso lingüístico y, a partir de este diagnóstico, diseñar una propuesta didáctica para su enseñanza en la educación superior.

En cuanto a la metodología, desde el punto de vista cualitativo, realicé un análisis lógico-semántico de los conectores discursivos, que se hallaban entre proposiciones de un mismo párrafo y también entre proposiciones de transición; es decir, de un párrafo a otro, a partir de la tipología citada arriba y de los criterios de uso, de variedad y de matices de significado.

Para efectos de la presentación de los resultados, en un primer momento, expondré la variedad de conectores que empleó la totalidad de los estudiantes; en un segundo momento, trataré mediante ejemplos particulares algunas incidencias de uso a las cuales llamaré "habitar el país de Babel", en honor al desasosiego y, por ende, al esfuerzo de los lectores para interpretar el significado de la información que se pretende comunicar. En un tercer momento, comentaré los hallazgos, que encontré en el corpus lingüístico, en torno al criterio de matices de significado. 
Las incidencias particulares del uso de los conectores discursivos $\mathrm{y}$ algunos hallazgos referentes a los matices de significado los presentaré mediante tablas ${ }^{4}$, que incluirán los siguientes elementos: un título, que aludirá a la aparición del conector discursivo en el grupo de proposiciones en estudio y de un argumento (proposición que expresa una razón); el número de las proposiciones seleccionadas, provenientes de diez ensayos estudiantiles (todas las proposiciones de cada ensayo se enumeraron para identificar aquellas que contenían un conector discursivo) y las proposiciones mismas.

Así pues, dentro de los conectores aditivos y organizadores de la información discursiva que empleó el estudiantado se encontraron: también, tampoco, además, asimismo, incluso (inclusive), por otro lado, desde otro punto de vista, en primer lugar, en adición, en conclusión y a su vez, lo que indica una variedad moderada de esta clase de conector.

En general, se desprendió que el uso de conectores fue adecuado, según los postulados de Montolío (2001), pese a que su frecuencia fue exigua. La mayoría de las conexiones efectuadas solo unieron enunciados. Para iniciar con las incidencias particulares del uso de este tipo de conector, observen la siguiente tabla:

Tabla 1: Aparición de conector aditivo y organizador de la información discursiva y de argumento

\begin{tabular}{|c|l|}
\hline $\mathbf{1 4}$ & \multicolumn{1}{|c|}{ Proposiciones enumeradas } \\
\hline $\mathbf{1 5}$ & $\begin{array}{l}\text { También, tiene poca credulidad el Ministerio de Obras Públicas y } \\
\text { Transportes }\end{array}$ \\
\hline
\end{tabular}

Notemos cómo en el argumento de la proposición 14 se introduce mediante el conector aditivo y organizador de la información discursiva también, que suma información en un sentido positivo. Luego, observen la Tabla 2:

4 Estas tablas constituyen una adaptación de las tablas originales, que se hallan en el trabajo Propuesta didáctica para la enseñanza estratégica de los conectores discursivos utilizados en la producción del ensayo académico escrito por los estudiantes de primer ingreso, matriculados en el curso Expresión Oral y Escrita del Centro de Estudios Generales de la Universidad Nacional, durante el II ciclo del 2012; no obstante, ambas tablas contienen los mismos elementos. 
Tabla 2: Aparición de conector aditivo y organizador de la información discursiva y de argumento

\begin{tabular}{|c|l|}
\hline 32 & $\begin{array}{l}\text { En conclusión, este proyecto fue una muestra más de la falta de } \\
\text { planeamiento e irresponsabilidad por parte de las autoridades cor- } \\
\text { respondientes ya mencionadas, }\end{array}$ \\
\hline
\end{tabular}

En esta tabla se revela el marcador de cierre en conclusión, en el segmento textual 32, cuya misión es indicar el término de un grupo proposicional, mediante la recapitulación de ideas preliminares. Cabe destacar que este segundo ejemplo representa las pocas veces que el estudiantado organizó el discurso por medio de esta clase de conector.

¿Qué fue lo que sucedió? ¿Por qué no hubo continuidad en su uso? Creo que los estudiantes dispusieron, únicamente, de estos conectores porque son comunes en el intercambio lingüístico diario, de ahí que la mayoría de los catalogados como cultos estén ausentes. Asimismo, el hecho de que casi no hayan utilizado conectores para abrir, continuar y cerrar series discursivas indica que no ha habido un entrenamiento lógico-semántico respecto de los matices de significado (expositivos-argumentativos) y, por ello, se desconocen las implicaciones de usar uno u otro en un escrito formal.

En lo concerniente a los conectores contraargumentativos, los estudiantes recurrieron a esta lista: pero, sin embargo, no obstante, al contrario, si bien, aunque, a pesar de, aun así, pese a y aun cuando. Cuando revisé los ensayos estudiantiles, observé, en algunos casos, un deficiente empleo, ya que los utilizaron sin una intención contraargumentativa o los incorporaron en combinaciones contradictorias, desde la semántica. Veámoslo en la Tabla 3: 
Tabla 3: Aparición de conector contraargumentativo, conector aditivo y organizador de la información discursiva

\begin{tabular}{|c|l|}
\hline $\mathbf{3 1}$ & \multicolumn{1}{|c|}{ Proposiciones enumeradas } \\
\hline $\mathbf{3 2}$ & $\begin{array}{l}\text { cuando han sido este y los últimos gobiernos de la oligarquía los } \\
\text { campeones en la depredación y entrega de los recursos naturales. } \\
\text { pasados el Gobierno emitió un comunicado bajo el título "Posición } \\
\text { del Gobierno de la República en relación a la Ruta 1856". }\end{array}$ \\
\hline
\end{tabular}

En este ejemplo es notable comentar la función del conector pero dentro de la combinación vinculatoria pero además en la proposición 32 , combinación frecuente en el uso de la lengua. Si se retoma el contenido de la proposición 31, se cae en cuenta de que el conector pero debería expresar una contraposición de ideas en la proposición 32 y no es el caso, ya que en ambas proposiciones se aprecia información de la misma naturaleza semántica (argumentos negativos sobre el tema por desarrollar); por tanto, el uso de este conector es inapropiado. Siguiendo la misma línea temática, veamos la Tabla 4:

Tabla 4: Aparición de argumento y conector contraargumentativo

\begin{tabular}{|c|l|}
\hline & \multicolumn{1}{c|}{ Proposiciones enumeradas } \\
\hline $\mathbf{2 5}$ & El Gobierno costarricense tiene fallas como cualquier otro sistema, \\
\hline $\mathbf{2 6}$ & pero la corrupción ayuda a notarlas mucho más, \\
\hline $\mathbf{2 7}$ & no obstante, lo más preocupante es la repercusión negativa \\
\hline $\mathbf{2 8}$ & $\begin{array}{l}\text { que esta construcción de carretera ha desarrollado como una parte } \\
\text { de la cadena de acciones similares ocurridas en el pasado, el lla- } \\
\text { mado abstencionismo en las elecciones. }\end{array}$ \\
\hline
\end{tabular}

En la proposición 25 se muestra un argumento seguido de dos proposiciones que tienen por objetivo rechazarlo mediante los conectores pero y no obstante en las proposiciones 26 y 27 . Nótese que, en este sentido, no es posible, desde un punto de vista lógico-semántico, tener una secuencia de dos proposiciones, cuya función es el contraste de ideas, pues si ambas apuntan al mismo objetivo, ¿Cómo se llevará a cabo dicha contraposición?

Podría deducirse, grosso modo, que el uso inapropiado de esta clase de conector se debió al descuido del contenido lógico-semántico 
de los enunciados previos a los conectores contraargumentativos y a la interferencia del registro coloquial de la lengua en el formal, ya que en el primero se llevan a cabo deficientes combinaciones de conectores, que son inválidas semánticamente.

En lo tocante a los matices de significado, el mismo ejemplo de la tabla 4 señala un desconocimiento de estos, pues es redundante emplear dos conectores con un matiz de significado fuerte, desde la óptica argumentativa; no obstante, rescato el ejemplo de la Tabla 5 que denota un buen empleo:

Tabla 5: Aparición de argumento y conector contraargumentativo

\begin{tabular}{|l|l|}
\hline & \multicolumn{1}{|c|}{ Proposiciones enumeradas } \\
\hline $\mathbf{2 7}$ & Es un disparate pensar \\
\hline $\mathbf{2 8}$ & que el MOPT pueda con todo esta responsabilidad, \\
\hline $\mathbf{2 9}$ & cuando ya ha demostrado durante muchos años su ineficiencia, \\
\hline $\mathbf{3 0}$ & $\begin{array}{l}\text { elaborando carreteras con planos para una flota vehicular de hace } \\
\text { muchos años, }\end{array}$ \\
\hline $\mathbf{3 1}$ & Situación que ha cambiado considerablemente. \\
\hline $\mathbf{3 2}$ & $\begin{array}{l}\text { Sin embargo, a pesar de saber la gran deficiencia de dicha } \\
\text { institución, se le confió el proyecto }\end{array}$ \\
\hline
\end{tabular}

En la proposición 32, la estudiante emplea el conector contraargumentativo sin embargo, seguido del conector contraargumentativo $a$ pesar de, hecho apropiado porque el conector sin embargo, que introduce argumentos fuertes, privilegia parte del contenido de la proposición 32 (se le confió el proyecto), con lo cual se anula el contenido de las proposiciones 27, 28, 29, 30, 31 y el fragmento restante de la 32 (la gran deficiencia de dicha institución), en virtud de que, semánticamente, se adhieren al conector a pesar de, que encabeza argumentos más bien débiles, que no impiden la consecución del argumento fuerte.

En relación con los conectores consecutivos utilizados por los estudiantes, se destacaron: debido a, puesto que, gracias a, de esta manera, ya que, así, pues, a causa de, de esa manera y porque, cuyo empleo fue apropiado, según los axiomas de Montolío (2001), pues, en verdad, sustentaron argumentos dentro de un fragmento anterior o posterior. En este sentido, examinemos la Tabla 6: 
Tabla 6: Aparición de argumento y conector consecutivo

\begin{tabular}{|l|l|}
\hline & \multicolumn{1}{|c|}{ Proposiciones enumeradas } \\
\hline $\mathbf{2 5}$ & Es importante llevar desarrollo a cada rincón de nuestro país, \\
\hline $\mathbf{2 6}$ & garantizando servicios básicos como agua potable, salud y educación, \\
\hline $\mathbf{2 7}$ & el desarrollo es un proyecto país, \\
\hline $\mathbf{2 8}$ & que se debe planificar en armonía con el ambiente, \\
\hline $\mathbf{2 9}$ & pues, al fin y al cabo somos país \\
\hline $\mathbf{3 0}$ & que dependemos del turismo \\
\hline $\mathbf{3 1}$ & y nos jactamos de democracia consolidada. \\
\hline
\end{tabular}

Aquí, se percibe un argumento en la proposición 25 que asegura su validez argumentativa por medio de leyes de paso $^{5}$ en la proposiciones 26, 27 y 28, al instaurarse como causas, que se suplementan mediante el conector consecutivo pues, el cual introduce las consecuencias relativas a estas en las proposiciones 30 y 31 . De este modo, es notoria la edificación, por parte del estudiante, de una relación de causa-efecto, de cara a organizar la conexión de ideas dentro de un párrafo.

No obstante, una minoría de estudiantes los utilizaron para contraponer ideas, hecho que no tiene sentido desde una perspectiva lógico-semántica, como se aprecia en la Tabla 7.

Tabla 7: Aparición de argumento y conector consecutivo

\begin{tabular}{|c|l|}
\hline & \multicolumn{1}{|c|}{ Proposiciones enumeradas } \\
\hline $\mathbf{4}$ & El mismo Estado pierde millones de colones en multas, \\
\hline $\mathbf{5}$ & al no hacer acatar en una forma correcta las leyes de tránsito \\
\hline $\mathbf{6}$ & y de esa manera cumplir con su deber y función. \\
\hline
\end{tabular}

En la proposición 4, se tiene la presencia de un argumento, seguido de una ley de paso en la proposición 5, hecho que debería consolidarse con el uso del conector consecutivo de esa manera, en la proposición 6 , el cual explicita una causa anterior a él, gracias al valor anafórico del pronombre esa. Sin embargo, el contenido de la proposición 6 se opone al de la proposición 5, desde un punto de vista lógico-semántico, por lo tanto, no se establece una relación de causa y efecto. De ahí que para el estudiante

5 La ley de paso es un argumento, cuya función es transferir a la conclusión la aceptación que se le atribuye a tal argumento (Plantin, C.:2008, p. 42 ). 
las pérdidas millonarias del Estado costarricense en cuanto a multas son resultado de la incapacidad de no hacer acatar a los conductores las leyes de tránsito. A lo anterior, considero que este par de ideas no poseen relación lógica entre sí, puesto que el Estado recopila dinero a través de multas, gracias a las infracciones de las leyes de tránsito y no en el caso contrario.

Respecto de los matices de significado, la mayoría de los estudiantes se inclinó por el uso de conectores causales (porque, ya que, puesto que, visto que, puesto que, dado que, debido a que), lo cual me indica que se debería tratar con atención el aprendizaje de los conectores consecutivos que provienen de una causa (por eso, por ello o por esa/ tal/dicha/razón/ causa/ese motivo, de ahí que, por lo que) y los conectores consecutivos que se enfocan en la consecuencia (en consecuencia, por consiguiente, por tanto).

Pese a que este tipo de conector es el más frecuente y el mejor utilizado por los estudiantes, creo que hay dos grandes objetivos por cumplir en el aula: el aumento de la variedad de este, lo cual debería aprovecharse para propiciar la concientización de sus matices de significado.

Por último, el análisis de los conectores de finalidad me reveló que el único conector que los estudiantes usaron fue para (que), con el propósito de determinar la causa como meta del enunciado, tal como lo señala Calsamiglia y Tusón (2007). Lo anterior, se demuestra en la siguiente tabla:

\section{Tabla 8: Aparición de argumento y conector de finalidad}

\begin{tabular}{|c|l|}
\hline & \multicolumn{1}{|c|}{ Proposiciones enumeradas } \\
\hline $\mathbf{4 4}$ & Una construcción gigantesca sin controles con el pretexto de emergencia \\
\hline $\mathbf{4 5}$ & para no brindar información "reveladora". \\
\hline $\mathbf{5 0}$ & $\begin{array}{l}\text { Como para cerrar con broche de oro, una declaratoria de "secreto de } \\
\text { estado". Sin derecho a confirmar el "chorizo". Un negocio perfecto. }\end{array}$ \\
\hline
\end{tabular}

A pesar del uso acertado de este conector, resta trabajar con los estudiantes, a fin de que extiendan su variedad. 


\section{Algunos aspectos de una propuesta didáctica para la enseñanza estratégica de los conectores discursivos significaría el éxodo del país de Babel}

El propósito del Trabajo Final de Graduación, para optar por el grado de Magister en Educación con énfasis en Docencia Universitaria, en la Universidad Nacional, consistió en diseñar una propuesta didáctica, cuyo objetivo es proveerles a los docentes universitarios, del área de lingüística, una serie de recursos didácticos para la enseñanza de los conectores discursivos en el registro formal. De este objetivo se desprenden tres específicos: incentivar el uso de los conectores discursivos, incrementar su variedad e impartir sus matices de significado, de manera que los estudiantes logren apropiarse de este recurso lingüístico y escriban textos cohesionados; en otras palabras, que abandonen el "país de Babel".

No obstante, en este ensayo, solo describiré, grosso modo, cada una de las fases de la enseñanza estratégica y luego, ilustraré la aplicación de dichas fases a través de un método o de una actividad, desde la arista del incremento de la variedad de conectores discursivos.

Ahora bien, si se habla de enseñanza estratégica, creo indispensable definir qué se entiende por estrategia. Para Monereo (2001), en el ámbito educativo, la estrategia constituye la toma de decisiones intencional, consciente y contextualizada, a fin de alcanzar los objetivos de enseñanza planificados por el docente, de forma que el estudiantado sepa operacionalizar los pasos por seguir en determinado procedimiento y discriminar cuándo y por qué la estrategia seleccionada es pertinente en ese contexto específico.

Entonces, ¿Cómo echar a andar la enseñanza estratégica? Esta se organiza en secuencias didácticas presentadas en tres fases, que a su vez, se desarrollan por medio de distintos métodos o actividades; todo ello, para garantizar el cumplimiento en el contexto de aula.

La primera fase se denomina Presentación de la estrategia. Consiste en una serie de pasos y de decisiones, que orientarán la resolución de un problema específico (demanda del profesor a los estudiantes), desde el inicio hasta el fin. En ella, el docente debe externar, con detalle, los pasos y las decisiones que entran en juego durante la resolución del problema, con el objeto de que el estudiantado pueda, lentamente, incorporarlos a su universo cognitivo. 
La segunda fase se llama Práctica guiada de la estrategia. Aquí, el estudiantado practica la estrategia modelada en la fase anterior; por su parte, el profesor mantendrá el control, especialmente, en las primeras aplicaciones. En consecuencia, es recomendable que estas actividades sean similares a las que se emplearon en la Presentación de la estrategia. Poco a poco, el docente podrá incluir cambios medulares, a fin de observar cómo los estudiantes toman decisiones y varían la estrategia, según el contexto de resolución.

La tercera fase tiene por nombre Práctica autónoma de la estrategia. Una vez que los estudiantes hayan interiorizado la estrategia seleccionada, para resolver la demanda de aprendizaje, estos deberían reproducirla en nuevas y parecidas situaciones de aprendizaje. Esta estrategia se materializa en un conjunto de preguntas, que los estudiantes deben responderse a sí mismos sobre la planificación y la puesta en práctica de la toma de decisiones.

Así las cosas, para ejemplificar la primera fase, me referiré al método Modelado metacognitivo ¿Cuál es su importancia? La escritura formal es un proceso cognitivo, que acontece en la mente del escritor. Como los lectores solo pueden acceder al resultado de ese proceso, al leer un texto nunca comprenderán cómo se construyó y por qué se eligió una determinada secuencia de ideas en vez de otra.

En el ámbito de la enseñanza de los conectores discursivos, el uso del Modelado metacognitivo devela este proceso silencioso e individual de la vinculación de ideas, con la finalidad de descubrir las decisiones que suscitaron procedimientos en la mente del docente-escritor y así ejecutar con el estudiante-lector una reflexión consciente sobre el desarrollo del proceso.

Para ello, el docente expone en la pizarra un texto que él haya escrito, de cuatro cinco párrafos, en los cuales se evidencien diversas conexiones de ideas. Durante esta actividad, el docente lee los párrafos, en voz alta, y explica, detalladamente, cuál fue la planificación de escritura, de dónde tomó la información para construir los párrafos, cuáles fueron los conectores discursivos que empleó, cuál es su funcionalidad y por qué los considera oportunos en la configuración de dicho texto.

En la segunda fase, el docente ha socializado la estrategia seleccionada para llevar a cabo conexiones lógico-semánticas entre proposiciones de un mismo párrafo o entre párrafos, al elaborar un texto; por ello, es de esperar que el estudiantado se valga de una estrategia similar. En 
la formalización de esta fase, el docente podría valerse del aprendizaje cooperativo mediante la actividad llamada Dentro y fuera del círculo.

¿Cómo hacerlo? El estudiantado forma dos círculos concéntricos, en los cuales los integrantes se miran unos a otros, al formar parejas. En cada una de la rondas, el docente les distribuirá proposiciones o párrafos, sin conectores, que ameriten llenarse con diferentes relaciones lógico-semánticas, para que cada pareja los complete, según corresponda.

Posteriormente, se procede a la discusión en grupo, con el fin de valorar si hubo una actuación estratégica por parte del estudiantado; es decir, si fue capaz de elegir lo que fue necesario, si lo supo hacer y por qué lo hizo de esa manera.

Ya en la tercera fase, se esperaría que el estudiantado pueda seleccionar y planificar una estrategia, en nuestro caso, elegir los conectores necesarios para articular proposiciones o párrafos según las operaciones semánticas en cuestión; todo ello, en virtud de que el docente en las dos fases previas ha estimulado, poco a poco, la autonomía en la actuación del estudiante.

Un método para lograr el cometido de esta fase es la Revisión de la estrategia de resolución, en el cual el estudiantado planifica por escrito una estrategia, que le ayudará a escribir un párrafo expositivo y otro argumentativo, con variedad de conectores, sobre un tema libre. Luego, el grupo de estudiantes deberá ejecutarla al desarrollar la demanda de escritura y, por último, se revisa entre los compañeros, para introducirle cambios, si fuera indispensable.

Terminada la actividad, el docente debería aprovechar la realimentación entre pares, a fin de juzgar la eficacia de la estrategia, en una plenaria o en entrevistas y, de no ser así, motivar con su asesoría el diseño de nuevas estrategias, para emplearlas en futuros contextos similares (Monereo, 2001).

En síntesis, después de haber analizado el desempeño del estudiantado universitario respecto de los conectores discursivos, prefiero hablar de altibajos discursivos y no de errores discursivos, ya que el uso de este recurso lingüístico es irregular, en el sentido de que unas veces los utilizan, de forma adecuada, aunque no haya variedad; otras veces, inadecuada, y esto también oscila dentro de un mismo tipo de conector, en vista de que no hay aprendizaje sobre el empleo de los matices de significado, quizás solo una intuición, para jerarquizar ideas en puntos estratégicos de secuencias textuales expositivas o argumentativas. 


\section{Conclusión}

Al igual que los investigadores mencionados al inicio del ensayo (Rojas y Umaña; 1986; Jiménez, 1987; Quesada y Ramírez, 1993; Garita, 2001 citados por Sánchez, 2006), estoy convencida de que la educación formal nos ha ido transformando en analfabetas discursivos, de forma que sabemos identificar y clasificar palabras; así como analizar sintácticamente una oración, pero no podemos, con facilidad, construir párrafos cohesionados, de manera estratégica.

Si desde el punto de vista de Freire (Sánchez, 2007), la educación solo es válida en la medida que propicia en el sujeto una conciencia crítica y si, al decir de Cassany (1999), la escritura contribuye al progreso de capacidades intelectuales, no solo porque exige análisis, reflexión, originalidad, objetividad, síntesis y distinción entre datos, sino también porque aflora en el escritor opiniones e ideas, que no se habían concebido antes de escribir, la escrituralidad constituye una poderosa herramienta de generación de conocimiento, en el entorno educativo, que debemos explotar los docentes, a fin de potenciar en el estudiantado dos tipos de competencias: las cognitivas, asociadas a los pensamientos comprensivo, crítico y creativo $^{6}$, en el transcurso de una demanda de escritura, y las metacognitivas, las cuales facilitan los procesos de toma de decisiones y de solución de problemas, al ser cimientos de la enseñanza estratégica.

6 Según Sanz (2010) el pensamiento comprensivo comprende, clarifica e interpreta información mediante el análisis, la síntesis, la clasificación, la comparación y el descubrimiento de razones; el pensamiento crítico examina e infiere la solidez de ideas y la validez de acciones para emitir juicios por medio de la investigación de la fiabilidad de fuentes, la interpretación de causas, la predicción de efectos y los razonamientos inductivo y deductivo; el pensamiento crítico es la capacidad de generar ideas y combinarlas de forma novedosa, a través del establecimiento de relaciones, producción de imágenes y de metáforas (pp. 41-68). 


\section{Referencias bibliográficas}

Alonso, P. (2013). Propuesta didáctica para la enseñanza estratégica de los conectores discursivos utilizados en la producción del ensayo académico escrito por los estudiantes de primer ingreso, matriculados en el curso Expresión Oral y Escrita del Centro de Estudios Generales de la Universidad Nacional, durante el II ciclo del 2012 (Tesis de maestría no publicada). Universidad Nacional, Heredia, Costa Rica.

Calsamiglia, H. y Tusón, A. (2007). Las cosas del decir: Manual de análisis del discurso (2da reimpresión). Barcelona: Ariel.

Cassany, D. (2008). Describir el escribir (6ta reimpresión). Buenos Aires: Paidós.

Cassany, D. (1999). Construir la escritura. Madrid: Paidós.

Cervera, Á., Hernández, G., Pichardo, C. y Sánchez, J. (2006). Saber escribir. Madrid: Santillana Ediciones Generales S.L.

Freire, P. (2007). Tres etapas en la evolución de la conciencia. En S. Sánchez (Ed.), Una pedagogía para el adulto (pp. 13-17). Buenos Aires: Espacio Editorial.

Montolío, E. (2001). Conectores de la lengua escrita. Barcelona: Ariel.

Monereo, C. (2001). La enseñanza estratégica. Enseñar para la autonomía. AULA. De Innovación Educativa, 100, 13-16.

Plantin, C. (2008). La argumentación (2da reimpresión). Barcelona: Ariel Practicum.

Sánchez, C. (2004). Historiografía de la enseñanza de la redacción en Costa Rica: los libros de texto. Revista de Filología y Lingüística, 30 (1), 219-246. Recuperado de http://www.latindex.ucr.ac.cr/filologia-30-1/14-SANCHEZ.pdf

Sánchez, C. (2006). Historia de un desencuentro: investigación y enseñanza de la redacción en Costa Rica. Revista de Filología y Lingüística, 32 (1), 223-245. Recuperado de http://www.latindex.ucr.ac.cr/filologia-32-1/13-SANCHEZ-223-245.pdf

Sanz, M. (2010). Competencias cognitivas en Educación Superior. Madrid: Narcea, S.A. De ediciones.

Silveira, M. I. M. (1999). Las principales concepciones de leguaje y sus postulados básicos. En: Línguas estrangeiras: uma visão histórica das abordagens, métodos e técnicas de ensino. Maceió-São Paulo: Catavento. Traducido por Ma. Inez Matoso. Recuperado de http:/www.google.es/\#hl=es\&xhr=t\&q=as+principales + concepciones + de + leguaje $+\mathrm{y}+$ sus + postulados $+\mathrm{b} \% \mathrm{C} 3 \% \mathrm{~A} 1$ sicos $\&-$ $\mathrm{cp}=63 \& \mathrm{pf}=\mathrm{p} \&$ sclient $=\mathrm{psy} \&$ source $=\mathrm{hp} \& \mathrm{aq}=\mathrm{f} \& \mathrm{aq} \mathrm{i}=\& \mathrm{aql}=\& \mathrm{oq}=\mathrm{as}+$ principa les + concepciones + de + leguaje $+y+$ sus + postulados $+b \% C 3 \% A 1$ sicos $\& p b x=1 \&-$ bav $=$ on.2,or.r_gc.r_pw.\&fp=98544aa383d54f81\&biw $=1280 \&$ bih $=533$. 